\title{
Smart Health Monitoring System for Pregnant Women
}

\author{
Saniya Ansari, M.B. Ansari
}

\begin{abstract}
For pregnant ladies, various health parameters like ECG, blood pressure, SPO2 (stamina), respiration rate, blood glucose level, body temperature, etc. need to be monitored regularly and must be in a normal level. If the mother' health become critical then definitely it will affect the baby. Hence it is recommended by physicians to do routine checkups at primary stages of pregnancy. But in rural areas, due to unavailability of well-equipped hospital facilities and also people don't have awareness about their health which yields in abnormalities or creates critical issues. The presented paper summarizes, the available system and their strength and weakness and challenges in health monitoring of pregnant women. The proposed system is used to analyze various pregnancy biological factors like heart rate of pregnant women \& Fetus, changes in blood pressure, blood glucose level, temperature, and weight. The proposed system will help for the rapid decision making and treatment through the high speed medical data transfer to the physicians over mobile for consulting and remote medical examinations. These values are transferred to database, to upload on server which can be monitored from any corner of the world.

Keywords: Heath Monitoring, Pregnant Women, Internet of Things, Health monitoring, heart beat rate, SPO2, and temperature sensor
\end{abstract}

\section{INTRODUCTION}

Due to unavailability of hospitals in rural areas and longer distance required to travel, People are not really conscious about their health, for small injuries and routine check-ups. Due to this pregnant ladies from rural areas avoids their regular check-ups at the initial stage of pregnancy. Regular check-ups will help to reduce abnormal children birth and fetal mortality rate. During Pregnancy, every trimester will be considered of 14 weeks and overall pregnancy length will be of 42 weeks. During this period, there is possibility of various complication due to maternal sepsis, bleeding and variation in blood pressure which may results in gestational diabetes and weight gain during pregnancy. During pregnancy few women may face the problems of high blood pressure which is called as gestational hypertension which can impact on the mother's kidneys and other organs. It can also results in low birth weight and premature delivery. Due to high blood sugar levels it is possible that the baby may gain extra weight. Due to

Revised Manuscript Received on April 25, 2020.

* Correspondence Author

Saniya Ansari*, Department of E \& TC Engineering, Dr D Y Patil School Of Engineering, Savirtibai Phule Pune University, Pune, India. Email: ansari.saniya6@gmail.com

M B Ansari, Department of Computer Engineering, BCOER, Savirtibai Phule Pune University, Pune, India. Email: mukram.apps@gmail.com

(C) The Authors. Published by Blue Eyes Intelligence Engineering and Sciences Publication (BEIESP). This is an open access article under the CC BY-NC-ND license (http://creativecommons.org/licenses/by-nc-nd/4.0/) underweight, pregnant women are more susceptible to decrease in the amount of amniotic liquid and there is a greater risk of having a preterm birth. [4][6]. Travelling a long distance during pregnancy is not recommended by physician. So it would be easier and helpful for them if there is a facility for regular checkup in nearby primary health care centers to monitor various health parameters at primary health care center and then transmit these data to specialist staying at distance via internet. Various sensors are available to measure heart beat rate, body temperature, Respiration, SPO2, ECG which is transferred to the specialist at distant hospital. The specialist interprets the data he received and tells whether the patient's condition is normal or not.

\section{LITERATURE SURVEY}

Suman Kumar et.al has proposed architecture to monitor health of pregnant women including adaptive and real time monitoring. An integrated system of mobile devices, various body sensors, cloud and client of health care professionals is designed for heath monitoring system [1]. Anandhi Ramachandran.et.al has proposed theoretical and conceptual structure using wearable sensors in remote monitoring of high risk pregnancy for continuous monitoring of vital parameters of pregnant women through wearable sensors [2]. Jennifer Runkle.et.al. Has proposed survey use of wearable sensors for pregnancy health monitoring and various methods to improve the diagnosis, monitoring, and managing of pregnancy health [3]. Gayathri S.et.al. Has developed IoT based Pregnant Women Health Monitoring System who stays in home during post operational days. They used heart rate sensor, temperature sensor, MEMS sensor, wifi module and arduino to gather the data [4]. T.Saraswathiet.al. has presented system for remote patient monitoring in healthcare field to constantly monitor physiological parameters such as pulse rate, temperature. By using data mining approaches, the system is trained for vital sign data and the system is trained and learned by supervised learning [5]. Devi .L M.Rekha et.al described remote pulse and temperature observing utilizing IOT for home use by patients that are not in a basic condition which will be monitored by specialist. [6]. S.Subalakshmi.et.al. designed a system to screen biological changes from the pregnant women to detect the uterine contraction, heart rate, blood pressure etc [7]. Shruthi.T.et.al has presented Fetal Health Monitoring System to measure the necessary health parameters like heart beat rate, body temperature, Respiration, SPO2(stamina), ECG which can be analysed by the specialist at distant hospital for consultation[8]. Vaishnavi Aswar et.al. Implemented the system to reduce the response time for a medical emergency and monitored constantly the heart pulse rate and BP monitor. If BP goes low or high, suddenly the system will generate a beep sound and it will be notified to doctor [9].

Published By:

Blue Eyes Intelligence Engineering DOI: 10.35940/ijeat.D7114.049420

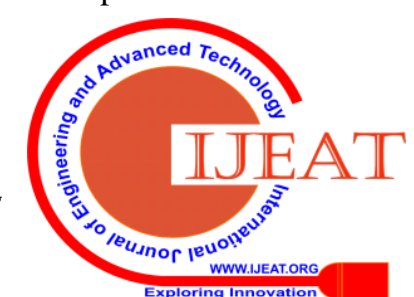


M.Nivedha.et.al. developed wearable healthcare device to monitor pregnant women health status continuously and provide timely alert. The device monitors the mother's physical activity, quality of sleep, heart rate and body temperature to analyze the patient's condition [10]. Nur Amira.et.al. Presented methodology for the fetal heart rate monitoring system. First the data is acquired using various sensors and after processing it is fed to microcontroller for analysis as per standard database then it's displayed \& sent to the remote doctor [11]. Sowmya S developed the system to measure the ECG, Heart rate, respiration rate and Dehydration analysis of both the mother and fetus using IoT and the values are sent to the concerned doctor for analysis. In case of any abnormality found by the doctor in the values, immediate actions are taken for the safety of the mother and fetus [12]. Joel J.P.C.et.al. Described a source of information for healthcare providers, specialists, and the general population interested in IOT [13]. P. Prabha.et.al [14] has proposed Prototype of a Wearable System for Remote Fetal Monitoring for pregnant ladies which measures Blood Pressure, Blood Sugar, Heart beat rate and the gathered information is send to the cloud [14].

A.C.Sumathi et.al described the design and implementation of wearable ECG in the form of smart shirt which can be used by any type of patient for monitoring his or her health in real time to get required treatment. These systems are mainly developed considering elder people in mind as they live alone in their homes [15]. S.Shiny Amala et.al [16] has proposed IoT Based compact assist device for monitoring various health parameters like temperature, heart rate of the maternal and the kick count of the foetus of Rural Pregnant Women to reduce maternal mortality. [16].

A.C.Sumathi,et.al the proposed system is used to measure some vital parameters of pregnant women, like temperature, heartbeat rate and kicking are measured [17]. V.Santhi et.al. Presented integrated system to monitor and measure parameters of pregnant women like pressure, temperature, heartbeat rate. In critical situation, the alarm will be raised and precautionary action will be taken [18]. S.Shiny Amalaet.al. Used an accelerometer sensor to measure the kick count of the fetus and the vital parameters such as temperature and heart beat using recent sensors and internet of things for personalized care [19]. E.C.Karvounis, et.al. Described an innovative, non-invasive remote monitoring of fetal and maternal vital signs. It consists of two modules: the wearable device and a centralized system [20] [21].

Padmavathi Narahari.et.al.[22] reported about the prevention measures for Anemia which is the most common nutritional deficiency disorder in the world and which decides the outcome of pregnancy [22]. C.K.Das.et.al. Described the development of heartbeat and temperature monitoring system based on a microcontroller which measures heartbeat and body temperature of a patient. Wireless system is used to transmit the measured data to a remote location [23]. Maria G. Signorini.et.al. Focused on monitoring procedures applied to fetal heart rate variability signals to assess fetal well-being. Their practicality is assessed by comparing normal fetuses and intra uterine growth restricted fetus. [24]. Mohamed Fezari et.al. Described the development of a heart rate monitor system based on a microcontroller in real-time. The system reads, stores and analyses the heart beat rate signals repetitively in real-time [25].
Ju Geon Pak and Kee Hyun's proposed a system using pulse oximeter to measure a patient's data and then it is transmitted to the personal monitoring server to analyze it by Physician. [26]. Pawan K. Baheti.et.al [26] described use of an ultra- low power pulse oximeter sensor, non-invasive monitoring of SpO2 and heart rate monitoring [27]. Amit S. Wale et.al. Presented a Heart Attack Prediction System using Neural Network and Genetic Algorithm. [28]. Mohamed Fezari.et.al.[30] described the development of a heart rate monitor system based on a microcontroller which reads, stores and analyses the heart beat rate signals repetitively in real-time [29]. M.Abdollahian.et.al. presented an approach to analyze and compare the hemoglobin level of pregnant women before and after consumption of Vitamin C and Sulfas Ferroses to evaluate the link between maternal hemoglobin level and pregnancy outcome [30]. V.Santhi, et.al. presented a framework to monitor the patient 's vibrant signs like EMG, Blood Pressure, Glucose Level, Bilirubin Count and the values will be sent to the database to update into the web server and to the doctor's mobile. It also enables the doctors to instantly send back their feedback to the nurse station. [31]. Adeline Boatin et.al. Presented the results of Verified functionality and acceptance of a wireless fetal monitoring technology in pregnant ladies. The experiment was tested in an inpatient labor unit with full-term singleton pregnancies. [32]. S.Subalakshmiet.al. Introduces a monitoring framework to screen physiological changes from the pregnant ladies. The attached sensors on patient's body to detect the uterine constriction, heart rate, blood pressure etc. It will recognize the unusual conditions and sends alert to the doctor [33].

\section{METHODOLOGY USED:}

In proposed system for pregnant ladies, some vital parameters of pregnant women, like blood pressure, hemoglobin level, temperature, heartbeat rate, blood pressure and kicking are measured to achieve the real time health monitoring of pregnant lady. This architecture of proposed system is shown in figure 3.1. It consists of various sensor units like heart rate detector, fetus heart rate detector, temperature sensor, oximeter, blood pressure, weight of fetus block, web portal section.

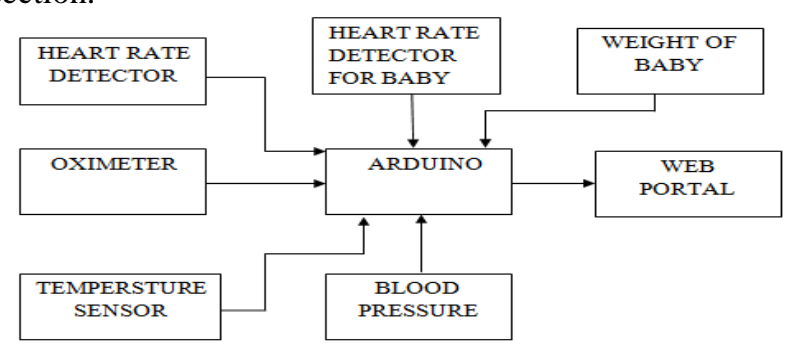

Fig 3.1: Smart health monitoring system for pregnant ladies

The heartbeat sensor (SEN-11574) is used to meaure the heartbeat for specific interval of time and Beats per Minute. It is usually clipped onto a fingertip or earlobe to get count of heart beat. Blood haemoglobin level sensor is used which gives a light of different wavelength for oximetry purpose. It used to compute blood oxygen level which is directly proportional to level of blood haemoglobin. For blood pressure calculation,

Published By:

Blue Eyes Intelligence Engineering

\& Sciences Publication 
differential transducer 015PDAA5 is used which is sensitive to air pressure that is given at the inlet at the top of IC. This blood pressure monitor measures the mean arterial pressure and approximates the systolic and diastolic pressures using a pressure transducer and an Arduino Uno. Oximeter sensor MAX30100 is used which gives the approx amount of blood oxygen. Pulse oximetry works on the absorption characteristics of red and IR light of oxygenated and deoxygenated hemoglobin. Blood oxygen concentration can be calculated from the ratio between the absorption red light and IR light by the hemoglobin. The change of blood volume is used to detect the Heart rate by the amount of light that passes through the finger. MAX30205 is used to measure Human Body Temperature. It converts temperature measurements to digital form using analog-to-digital converter (ADC). During prenatal visits and during labor, fetus heart rate can be observed using Doppler device to listen to and record baby's heartbeat through patient's belly (abdomen). In another way, the ultrasound probe (transducer) is fixed firmly to patient's belly which sends the sounds of baby's heart to a computer. Esp8266 wifi module is used for internet connectivity to provide the address of website in the code used. The ESP module continuously tries to establish a connection over internet to transfer the data from heart rate sensor, blood pressure, temperature sensor which was processed by Arduino board. Then the data is uploaded on server which can be monitored from any corner of the world.

\section{RESULTS:}

The below figure shows the results of various sensor outputs used for continuous health monitoring of pregnant women. Figure 4.1 shows the Simulation results of heart rate sensor when it is interfaced with arduino uno. Figure 4.2 display the Output of heart rate sensor on LCD screen and Figure 4.3 shows graph of continuous monitoring status of heart rate and blood pressure. Table 4.1 give the ideal Values of Various Sensors output which are used for evaluation purpose.

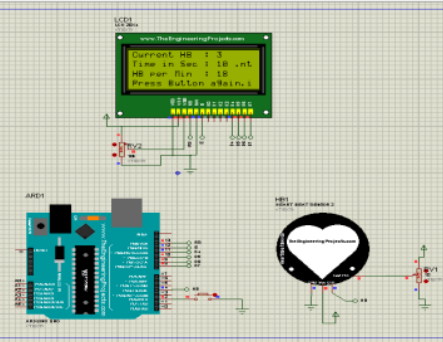

Fig.4.1 Simulation diagram of heart rate sensor interface with arduino uno

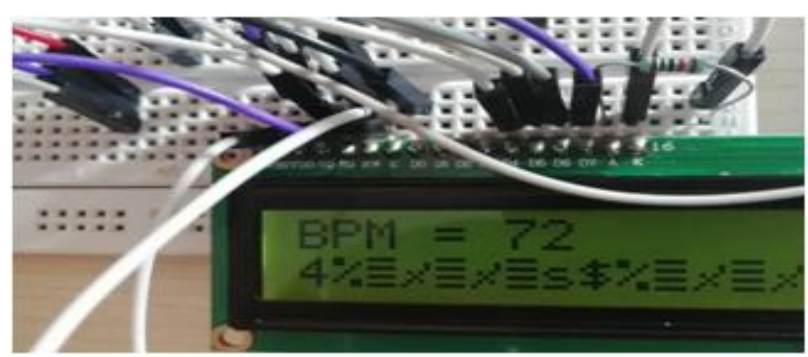

Fig.4.2 Output of heart rate sensor

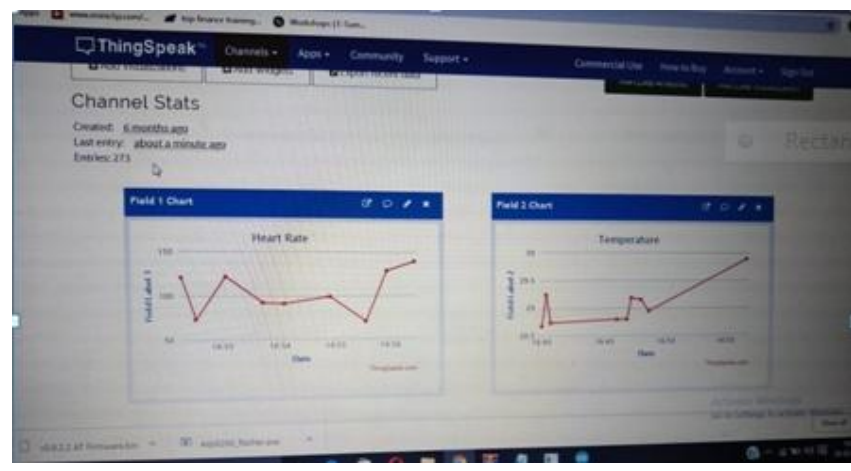

Fig 4.3 Results of heart rate and blood pressure monitoring

Table 4.1 Ideal Values of Various Sensors output

\begin{tabular}{|c|l|l|}
\hline Sr. No. & Parameter & \\
\hline \multirow{3}{*}{1} & Heart rate & \\
\cline { 2 - 3 } & Diastolic condition & 80 BPM \\
\cline { 2 - 3 } & Systolic condition & 120 BPM \\
\hline \multirow{2}{*}{2} & Temperature & \\
\cline { 2 - 3 } & Normal & $97 \mathrm{~F}-99 \mathrm{~F}$ \\
\cline { 2 - 3 } & High & $100.4 \mathrm{~F}$ \\
\hline \multirow{3}{*}{3} & Hemoglobin & \\
\cline { 2 - 3 } & Men & $14-17 \mathrm{gm} / \mathrm{dl}$ \\
\cline { 2 - 3 } & Women & $12-15 \mathrm{gm} / \mathrm{dl}$ \\
\hline
\end{tabular}

\section{CONCLUSION}

The existing system are not wearable and it occupies more space and are less accurate. The proposed system measure various parameters using sensory platform, then it sends the data to doctor. The proposed system is compact in nature and wearable. When the patients data will be received, , doctor analyzes it and provides suitable treatment. The proposed system will be helpful to pregnant ladies to avoid miscarriage and the Physicians can also suggest healthy diet. In proposed system vital health parameter such blood pressure, kicking, body temperature, heart rate, fetus heart rate are analyzed and report will be generated which can be used for observation.

\section{REFERENCES}

1. Suman Kumar, Yashi Guptay, Vijay Magoy, Health-monitoring of pregnant women: Design requirements, and proposed reference architecture,16th IEEE Annual Consumer Communications \& Networking Conference, USA, Lakehead University, Thunder Bay, ON P7B 5E1, Canada 2019

2. Ritika, Anandhi Ramachandran. "Wearable Sensors: A Step towards Smart Monitoring Of High Risk Pregnancies", International Journal Of Scientific \& Technology Research, VOLUME 8, ISSUE 02, FEBRUARY 2019 ISSN 2277-8616

3. Jennifer Runkle, Maggie Sugg, Danielle Boase, Shelley L. Galvin and Carol C. Coulson, "Use of wearable sensors for pregnancy health and environmental monitoring: Descriptive findings from the perspective of patients and providers", Digital Health Volume 5: 1-14, 2019, DOI: 10.1177/2055207619828220

4. Gayathri S,,Bharathi T, Devleena Jerusha AR, Ajay Kumar, “A Pregnant Women Health Care Monitoring System Based on IoT" IJETMS, Issue:1, Volume No.2, January 2018

5. T.Saraswathi,S.Amutha, "Iot Based Wireless Healthcare Monitoring", AIJR Publisher in Proceedings of the 3rd National Conference on Image Processing, Computing, Communication, Networking and Data Analytics, April 2018.

6. Devi .L M.Rekha, "Pregnant Women Healthcare Monitoring System Based On Iot",April 2018

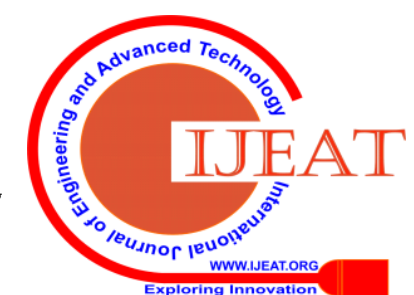


7. S. Subalakshmi, G. Murugaboopathi, D. Senthilkumar,"Efficient EER-LEACH Protocol for Monitoring the Activities of Pregnant Women Using Wearable Body Sensor Network",Volume 7,2018.

8. Shruthi.T, Sripriyaa.V.N, Suganthi.V, Kalpana.R, Kapila Vani.R.K, Fetal Health Monitoring System International Journal of Scientific Research and Review, ISSN NO: 2279-543X, Volume 7, Issue 3, 2018

9. Vaishnavi Aswar, Pratiksha Takawane, Umakant Jadhav, Pratik Bondre, " A Model of an Automatic Blood Pressure Monitoring and Triggering System for Hospital”, Vol. 6, Issue 9, September 2017.

10. M.Nivedha, J.P.Vinusha, Mrs. M.Saraswasthi, "Wearable Healthcare Device for Pregnant Women", Vol.9, No.1 April 2017.

11. Nur Amira Nadia, Binti Mohd Shabry, O.P. Singh, Pranshul Sardana, Rozana binti Hisham and M. B. Malarvili, "Home Based Fetal Heart Rate Monitor", Volume 12, Number 52017.

12. Sneha M, Sowmya S "Foetal Health Monitoring System", International Journal of Scientific \& Engineering Research Volume 8, Issue 5, May-2017 43 ISSN 2229-5518, May 2017.

13. Joel J. P. C. Rodrigues, Dante B. R. Segundo, Heres A. Junqueira , Murilo H. Sabino, Rafael M. Prince, "Enabling Technologies for the Internet of Health Things",January 2018,IEEE Access PP(99):1-1,DOI: 10.1109/ACCESS.2017.2789329, Nov 2017

14. P. Prabha, R. Priyadharsini, N. Priyanka, C. Ramya, G. Rajkumar, S. Mohanraj, M.Kumarasamy ISSN: 0974-2115 Journal of Chemical and Pharmaceutical Sciences, Special Issue 5: March 2017

15. A.C.Sumathi, P.Shayestha, M.Umira, S.Vinothini, "Health Monitoring System for Pregnant Women"

16. International Journal of P2P Network Trends and Technology, Volume 7 Issue 4 July to August 2017.

17. S.Shiny Amala, S.Mythili "IoT Based Health Care Monitoring System For Rural Pregnant Women" International Journal of Advanced Research in Electronics and Communication Engineering (IJARECE) Volume 6, Issue 11, November 2017.

18. A.C.Sumathi, P.Shayestha, M.Umira, S.Vinothini, "Health Monitoring System for Pregnant Women", Volume 7 Issue 4 July to August 2017.

19. V.Santhi,K.Ramya ,APJ.Tarana ,G.Vinitha "IOT Based Wearable Health Monitoring System for Pregnant Ladies Using CC3200",IJARMET, 3 May 2017.

20. S.Shiny Amala, S.Mythili, "IoT Based Health Care Monitoring System For Rural Pregnant Women", ISSN: 2278 - 909X, IJARECE, Volume 6 , Issue 11, November 2017.

21. E.C. Karvounis, C. Papaloukas, K. Papanikolaou and D.I. Fotiadis, "A Wearable Health Monitoring System During Pregnancy”, 23 November 2015.

22. Hyun Jung La, Sangdo-Ro, Dongjak-Gu, Han Ter Jung, and Soo Dong Kim, "Extensible Disease Diagnosis Cloud Platform with Medical Sensors and IoT Devices", 2015 3rd International Conference on Future Internet of Things and Cloud DOI: 10.1109/FiCloud.2015.65 June 2015.

23. Padmavathi Narahari,Hephzibah kirubamani, "Awareness of anemia in pregnancy among the caregivers of pregnant women in Saveetha Medical College Hospital", Volume 5, Issue 3, March 2015.

24. C. K. Das, M. W. Alam and M. I. Hoque, "A Wireless Heartbeat And Temperature Monitoring System For Remote Patients”, 1-3 May 2014.

25. Maria G. Signorini, Andrea Fanelli, Giovanni Magenes, "Monitoring Fetal Heart Rate during Pregnancy: Contributions from Advanced Signal Processing and Wearable Technology", Volume 2014.

26. Mohamed Fezari, Mounir Bousbia-Salah, and Mouldi Bedda, "Microcontroller Based Heart Rate Monitor" 2012.

27. Ju Geon Pak and Kee Hyun, Park, Sindang-dong, "Advanced Pulse Oximetry System for Remote Monitoring and Management”.2011.

28. Pawan K. Baheti, Harinath Garudadri, "An ultra-low power pulse oximeter sensor based on compressed sensing", 9 June 2009.

29. Amit S. Wale Shilpa S. Sonawani , Prof. Shridevi C. Karande , "ECG Signal Analysis And Prediction Of Heart Attack With The Help Of Optimized Neural Network Using Genetic Algorithm “, 2008

30. Mohamed Fezari, Mounir Bousbia-Salah, and Mouldi Bedda, "Microcontroller Based Heart Rate Monitor", Vol. 5, No. 4, October 2008.

31. M. Abdollahian , S. Ahmad, S. Nuryani ,D. Anggraini, “Assessment of Hemoglobin Level of Pregnant Women Before and After Iron Deficiency Treatment Using Nonparametric Statistics”, 2008.

32. Godavarthi Rajesh M.K. Srilekha, "Advanced Healthcare Monitoring System Using Cc3200 Microcontroller”, Volume 115 No. 82017 , 419-424.

33. Boatin, Blair Wylie, Ilona Goldfarb, Robin Azevedo, Elena Pitte,Courtney Ng, Jessica Haberer," "Wireless Fetal Heart Rate Monitoring in Inpatient Full-Term Pregnant Women:Testing Functionality and Acceptability Adeline A"." University,Coimbatore. Cloud Computing. EER-LEACH Protocol for Monitoring the Activities of Pregnant EER-LEACH Protocol for Monitoring the Acti
Women Using Wearable Body Sensor Network".

\section{AUTHORS PROFILE}

Dr. Saniya Ansari, Ph.D.(ECE), is currently working as Faculty of Dr D Y Patil School Of Engineering, Pune. The research interest Areas are Digital Image Processing, Robotics. She has comleted Ph.D. in ECE from Karpagam

Mr. Mukram Ansari, Assistant Professor, Compute Department is presently working in JSPM, Wagholi, 